\title{
CBIR of Batik Images using Micro Structure Descriptor on Android
}

\author{
Agus Eko Minarno', Yuda Munarko ${ }^{2}$, Arrie Kurniawardhani ${ }^{3}$ \\ ${ }^{1,2}$ Universitas Muhammadiyah Malang, Malang, Indonesia \\ ${ }^{3}$ Universitas Islam Indonesia, Yogyakarta, Indonesia
}

\begin{tabular}{l} 
Article Info \\
\hline Article history: \\
Received Feb 24, 2018 \\
Revised Apr 30, 2018 \\
Accepted May 7, 2018 \\
\hline
\end{tabular}

Keyword:

Android

Batik

CBIR

Micro-structure

\begin{abstract}
Batik is part of a culture that has long developed and known by the people of Indonesia and the world. However, the knowledge is only on the name of batik, not at a more detailed level, such as image characteristic and batik motifs. Batik motif is very diverse, different areas have their own motifs and patterns related to local customs and values. Therefore, it is important to introduce knowledge about batik motifs and patterns effectively and efficiently. So, we build CBIR batik using Micro-Structure Descriptor (MSD) method on Android platform. The data used consisted of 300 images with 50 classes with each class consists of six images. Performance test is held in three scenarios, which the data is divided as test data and data train, with the ratio of scenario 1 is $50 \%$ : $50 \%$, scenario 2 is $70 \%, 30 \%$, and scenario 3 is $80 \%: 20 \%$. The best results are generated by scenario 3 with precision valur $65.67 \%$ and recall value $65.80 \%$, which indicates that the use of MSD on the android platform for CBIR batik performs well.
\end{abstract}

Copyright () 2018 Institute of Advanced Engineering and Science. All rights reserved.

\section{Corresponding Author:}

Agus Eko Minarno,

Universitas Muhammadiyah Malang,

Jl. Raya Tlogomas 246 Malang, Indonesia.

(0341) 464318

Email: aguseko@umm.ac.id

\section{INTRODUCTION}

Batik is one of Indonesian culture that is recognized as world heritage. In terms of language, batik comes from the merger of two words namely "amba" which means area and "nitik" which means point. So, linguistically, batik is a series of points [1]-[3]. Indonesia has a wide range of batik motifs and patterns. In fact, batik motifs depict the characters and customs along with the noble values of each local area [4]-[7]. This knowledge needs to be introduced to the general public through an easily accessible application. Several studies on batik have been proposed, such as the Content Based Image Retrieval (CBIR) technique for retrieving batik images using Color Difference Histogram $(\mathrm{CDH})$ [8], enhanced micro-structure descriptor [9], Multi Texton Co-Occurrence Descriptor [10], Multi Structure Co-occurrence Descriptor [11], improved completed robust local binary pattern [12], Texture Fusion [13]. However, all those studies are build for desktop. In this study, a batik image retrieval application is tried to build in android platform.

Content Based Image Retrieval (CBIR) is one of active research nowdays, thus there are a lot of techniques proposed to seek the right method [14]-[17]. Techniques in CBIR, be it CDH and other techniques, such as texton co-occurrence matrix, multi-textons histogram, and micro-structure descriptor, depend on the feature extraction process [8]-[13]. Types of features can vary, such as color, texture, angle, and shape features. An example for the color feature is a gray level co-occurrence matrix (GLCM) that extracts features based on gray intensity by changing the image color to gray scale [18]-[20]. Further, an example for the edge feature is the use of the Sobel or Prewitt operator, which in some cases the Sobel operator provides better performance [21]-[23]. An example of feature texture is the use of texton proposed 
by Julesz [24]. In practice, CBIR often incorporates several types of features in order to obtain satisfactory results, for example merging texture and color features or edge and color features [12]. One of the combined feature extraction methods that has been proven to provide satisfactory results for CBIR batik is the MicroStructure Descriptor (MSD) [9]. Thus, MSD is chosen in this study to bulid a batik image retrieval application in android platform

\section{THE DATASET}

In this research, batik image dataset was collected through 50 types of batik cloth. For each type of fabric, six images were randomly photographed, then resized at 128x128 pixels in JPEG format. Thus, the total number of images in the dataset is 300 and consists of 50 classes. An example of a batik image is shown in Figure 1. In general, there are two patterns of batik drawings, geometric and non-geometric patterns [12]. Dataset is available download at https://github.com/agusekominarno/batik.
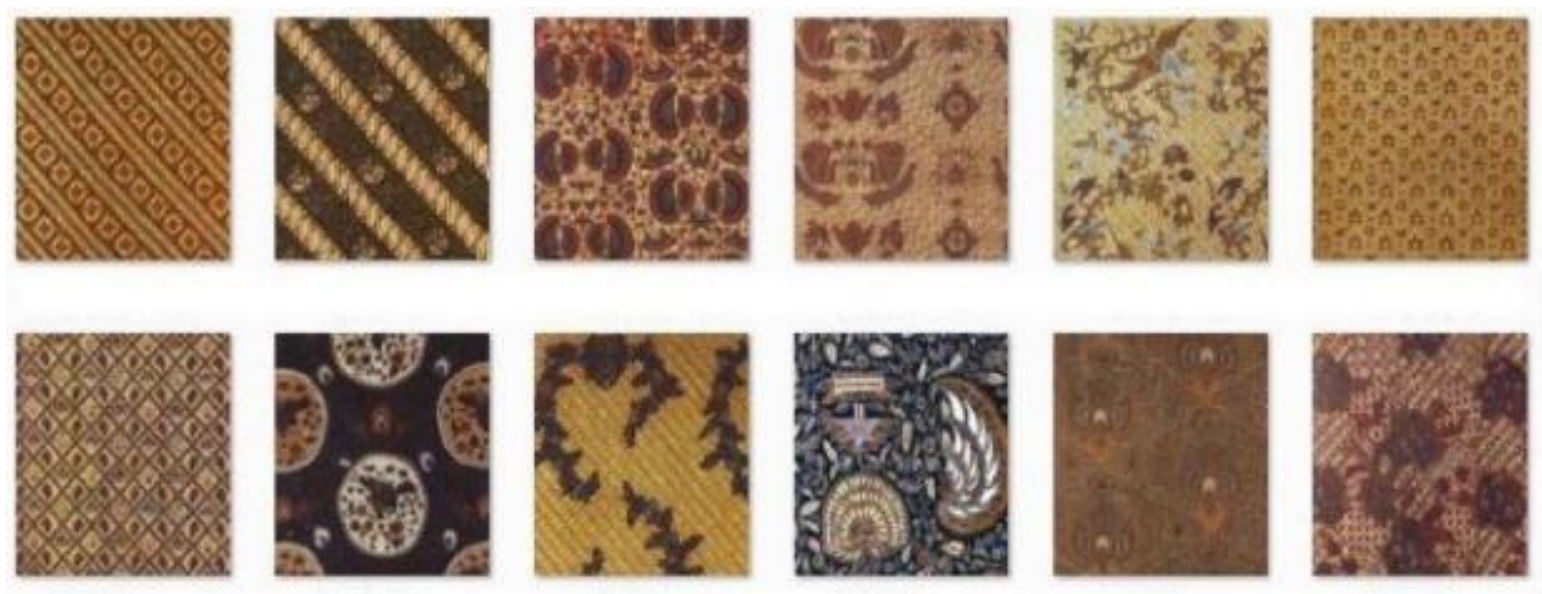

Figure 1. The example of batik images

\section{MICRO-STRUCTURE DESCRIPTOR (MSD)}

The extraction process used was Micro-Structure Descriptor method which is a feature extraction technique using texton. In this extraction, from each batik image, a histogram was generated which is a vector value for color features and edge orientation.

\subsection{The extraction of color feature}

The most popular color system is RGB, where RGB stands for red, green, blue. The colors formed on the RGB system are the result of a mixture of primary colors red, green, and blue, based on certain compositions. However, in the world of image processing, in addition to RGB there is HSV which is also popular. H in HSV is Hue which expresses the actual colors, such as red, violet, and yellow, which are used to determine redness, greenness, etc. Furthermore, S is Saturation, ie purity or color strength. Finally, the $\mathrm{V}$ for Value indicates the brightness of the color with a range of values between $0 \%$ to $100 \%$, where $0 \%$ will produce purely dark color, while the larger values will produce brighter colors and new variations of colors. In this study, the color system used is HSV, where the color was quantized to 72 colors with details of $\mathrm{H}$ as much as 3 bin, $\mathrm{S}$ as much as 3 bin, and $\mathrm{V}$ as much as 8 bin. Thus, the total color obtained is $3 \times 3 \times 8=72$ colors.

\subsection{The extraction of edge orientation feature}

The edge orientation has a strong influence on image perception. There are many methods for detecting edge features, in this research, edge detection was done with a sobel operator. This is because it has the ability to reduce noise before performing edge detection calculations. This capability provides better results when compared to gradient operators or other edge approach methods, so it is considered more efficient and simple [7].

The calculation on the sobel operator is done by weighing the pixels closer to the center of the window. Thus, the influence of neighboring pixels will differ according to their location to the point where the gradient is calculated. Gradient is the result of measurement of change in an intensity function and an image 
can be viewed as a collection of several continuous intensity functions of the image. The result of the sobel operator is then quantized to 18 bin, so that at this stage there will be 18 edge orientation features.

\subsection{Texton detection}

Figure 2 illustrates the process in MSD using texton. There are four types of texton, with a size of $3 \times 3$ grids, which are used to obtain micro-structure maps. As an example, let Figure 2(a) be the pixels of image with a quantized edge detection value. Using the existing textons, a scanning process of the image is performed. For each pattern similar to textons, the pixels are marked, shown in Figure 2(b). Figure 2(c) is the result of detection that already shows the micro-structure pattern. Figure 2(d) is a micro-structure map. Furthermore, in order to obtained histogram value, the micro-structure map is used as a mask to mark considered pixels. Only value from considered pixels are used to compose histogram. The illustration of the result is presented in Figure 3.

\begin{tabular}{|l|l|l|l|l|l|l|l|l|}
\hline 0 & 2 & 3 & 0 & 1 & 4 & 2 & 4 & 3 \\
\hline 1 & 2 & 4 & 4 & 1 & 0 & 2 & 4 & 1 \\
\hline 5 & 2 & 4 & 2 & 2 & 5 & 3 & 4 & 4 \\
\hline 3 & 3 & 1 & 4 & 1 & 4 & 6 & 6 & 1 \\
\hline 3 & 3 & 5 & 0 & 5 & 3 & 6 & 6 & 5 \\
\hline 1 & 2 & 0 & 1 & 5 & 0 & 1 & 5 & 0 \\
\hline 1 & 7 & 1 & 0 & 4 & 2 & 3 & 8 & 4 \\
\hline 1 & 7 & 3 & 1 & 3 & 2 & 2 & 8 & 2 \\
\hline 2 & 7 & 7 & 1 & 4 & 1 & 5 & 8 & 3 \\
\hline
\end{tabular}

(a)

\begin{tabular}{|l|l|l|l|l|l|l|l|l|}
\hline & 2 & & & 1 & & & 4 & \\
\hline & 2 & & & 1 & & & 4 & \\
\hline & 2 & & & & & & 4 & 4 \\
\hline 3 & 3 & & & 5 & & 6 & 6 & \\
\hline 3 & 3 & & & 5 & & 6 & 6 & \\
\hline & & & & 5 & & & & \\
\hline & 7 & & & & & & 8 & \\
\hline & 7 & & & & & & 8 & \\
\hline & 7 & 7 & & & & & 8 & \\
\hline
\end{tabular}

(c)

\begin{tabular}{|l|l|l|l|l|l|l|l|l|}
\hline 0 & 2 & 3 & 0 & 1 & 4 & 2 & 4 & 3 \\
\hline 1 & 2 & 4 & 4 & 1 & 0 & 2 & 4 & 1 \\
\hline 5 & 2 & 4 & 2 & 2 & 5 & 3 & 4 & 4 \\
\hline 3 & 3 & 1 & 4 & 5 & 4 & 6 & 6 & 1 \\
\hline 3 & 3 & 5 & 0 & 5 & 3 & 6 & 6 & 5 \\
\hline 1 & 2 & 0 & 1 & 5 & 0 & 1 & 5 & 0 \\
\hline 1 & 7 & 1 & 0 & 4 & 2 & 3 & 8 & 4 \\
\hline 1 & 7 & 3 & 1 & 3 & 2 & 2 & 8 & 2 \\
\hline 2 & 7 & 7 & 1 & 4 & 1 & 5 & 8 & 3 \\
\hline
\end{tabular}

(b)

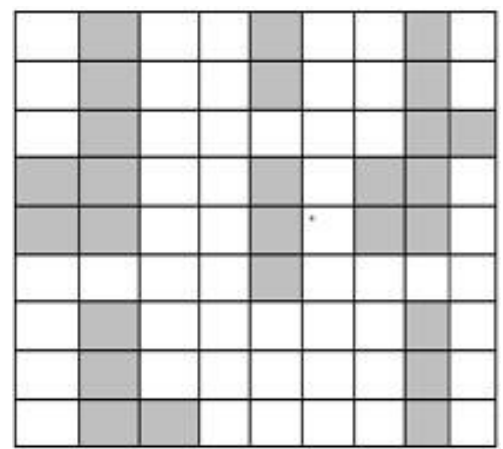

(d)

Figure 2. The illustration of texton detection

\subsection{Precision and recall} Equation (2):

In this study, performance was measured using the precision and recall defined in Equation (1) and

$$
\begin{aligned}
& \text { Precision }=\frac{i m g_{-} \text {retrieved_that_relevant }}{\text { img_retrieved }} \times 100 \\
& \text { Recall }=\frac{i m g \_r e t r i e v e d \_t h a t r e l e v a n t}{i m g \_r e l e v a n t \_ \text {in_collection }} \times 100
\end{aligned}
$$




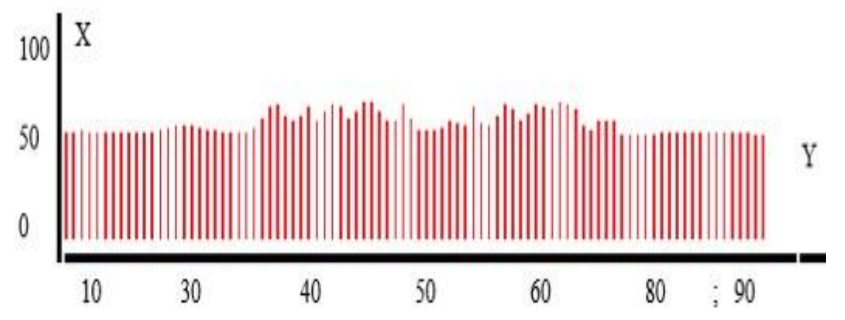

Figure 3. Merged histogram (color and edge)

\section{FEATURE IDENTIFICATION PROCESS (FEATURE EXTRACTION)}

As already mentioned, the method used for feature extraction is MSD. The series of processes of CBIR batik implementation with MSD is presented in Figure 4. The initial stage is to get the color feature by converting the RGB value to the HSV value. In addition, it also performed edge features extraction using the Sobel operator. The second stage is to quantify the value of HSV and edge orientation, so that obtained 72 color features and 18 edge orientation features. However, these features are not used directly, but look for the pattern of occurrences using textons, and resulting micro-structure map. Using this map, then the image pixels are masked. Only masked pixels are considered for histogram value, allowing 72 features related to color and 18 features related to edge orientation, so the total features obtained are 90 features.

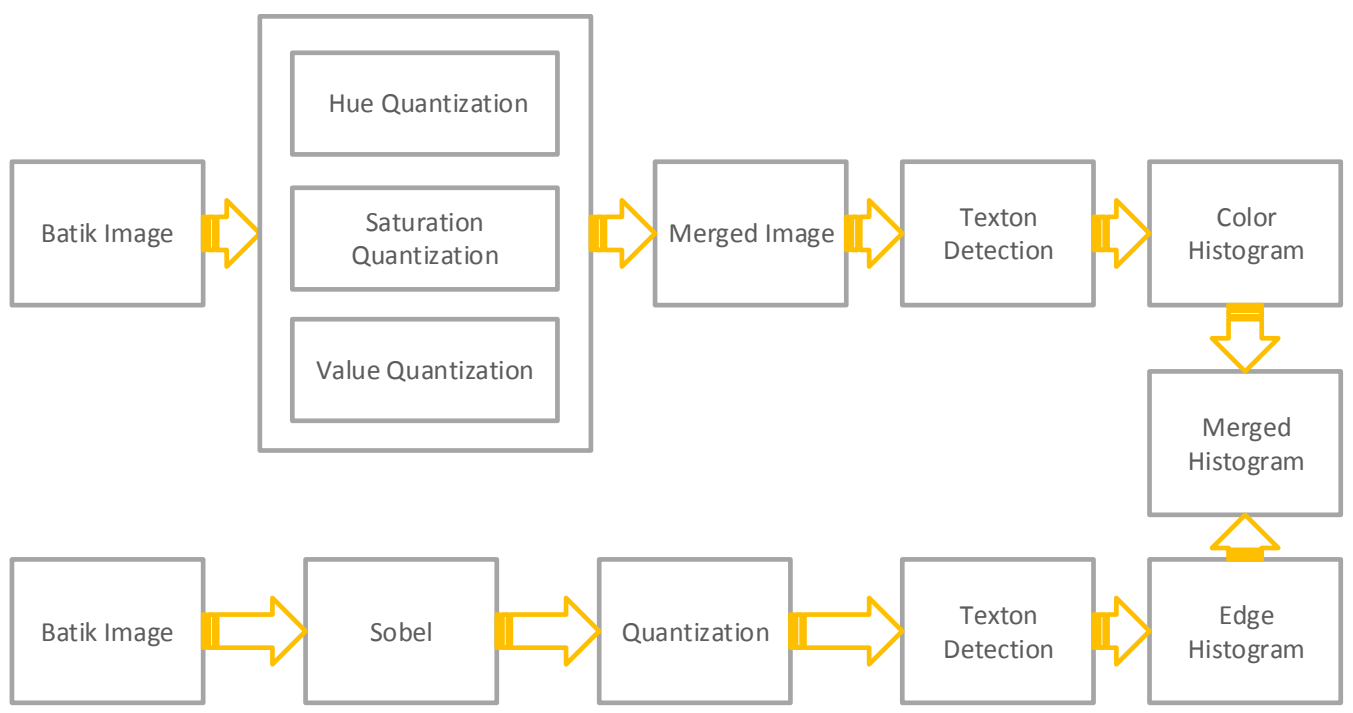

Figure 4. The Micro-structure description process

Figure 5 shows the initial step for color feature extraction process. The initial image used is the RGB image as shown in Figure 5(a). When converted from RGB to HSV, the image structure changes, the result can be seen in Figure 5(b). In the example image it can be seen that the Hue value tends to produce a red color, and the Saturation value decreases. After conversion, the value of HSV is quantized, where the value of $\mathrm{H}$ is divided into 3 bins, $\mathrm{S}$ becomes 3 bins, and $\mathrm{V}$ becomes 8 bins. The quantization results also cause a change in the appearance of the image, as shown in Figure 5(c). From this quantized image, a 72-vector histogram is obtained and presented as Figure 5(d), which furthermore represents as 72 color features.

Next, Figure 6 is the first step of the edge orientation feature extraction process. First, the image is converted to gray scale, shown in Figure 6a. Next, the edge orientation value is calculated using the Sobel operator, the result is shown in Figure 6(b). Last is the process of quantizing edge orientation values into 18 bin. The quantization results are shown in Figure 6(c). The extraction result in this process is a histogram with the number of vectors 18, shown in Figure 6(d). 


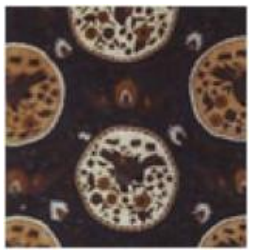

(a)

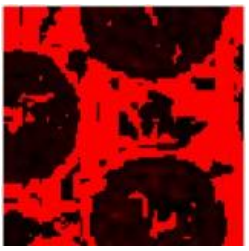

(b)

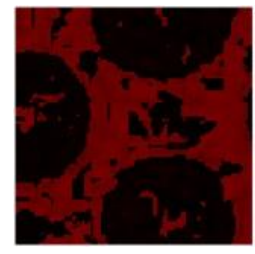

(c)

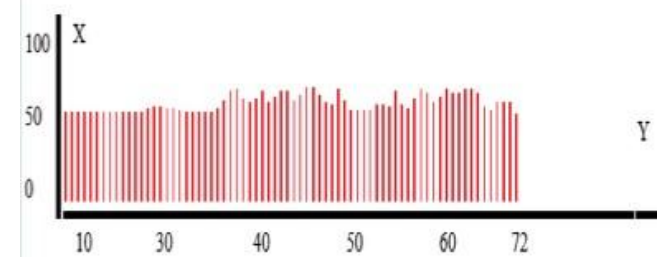

(d)

Figure 5. The feature extraction process

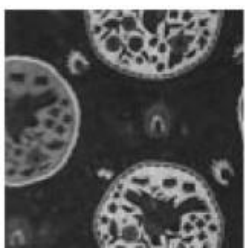

(a)

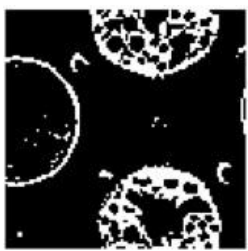

(b)

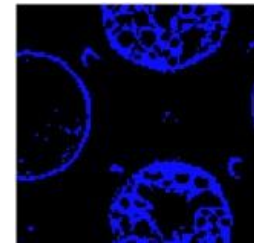

(c)

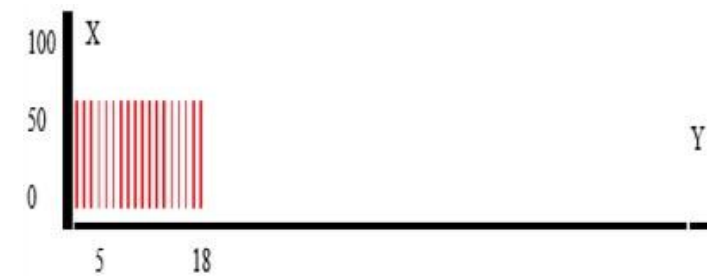

(d)

Figure 6. The feature extraction process

\section{RESULT AND ANALYSIS}

Testing was done by using precision and recall. Measurements were made to the images in the database containing 300 batik images classified into 50 classes, where each class consists of 6 images. There were three test scenarios, the first was to divide the image by 50\% of training data and $50 \%$ of test data, so for each class, 3 images were used as training data and 3 images as test data. The second scenario was to divide the images by $70 \%$ of training data and $30 \%$ of test data, where from each class there were 4 training images and 2 test images. The latter was to divide the data into $80 \%$ training data and $20 \%$ test data, therefore, for each class there were 5 images used as training data and 1 image as test data. Furthermore, the measurement of precision and recall values are performed on 6 retrievals, 8 retrievals, and 12 retrievals. The precision value is shown by Table 1, while the recall value is shown by Table 2 .

The features used in this CBIR are color and edge orientation features. However, these two types of features are not used directly, but, look for spatial correlation between the two features to get the histogram value. This special correlation was obtained by utilizing the MSD method which uses texton to form a mask or a micro-structure. Since there are basically two types of features used, then, the feature dimension used is 72 color features and 18 edge orientation features, so, totally, there are 90 features used.

The CBIR system is built on the android platform with input queries taken with the camera of the mobile device or retrieved from the database. In terms of execution time, to perform feature extraction process against query using MSD and to search for suitable batik image in the data base, it takes about 1 minute. In addition, the best performance shown by the test with the ratio of training data compared to the test data is $80 \%$ : $20 \%$, where the precision value is $65.67 \%$ and the recall value is $78.80 \%$.

Table 1. Precicion Rate

\begin{tabular}{lccc}
\hline & $50 \%: 50 \%$ & $70 \%: 30 \%$ & $80 \%: 20 \%$ \\
\hline 6 retrieval & $39.33 \%$ & $52.50 \%$ & $65.67 \%$ \\
8 retrieval & $30.00 \%$ & $40.38 \%$ & $50.75 \%$ \\
12 retrieval & $20.06 \%$ & $27.08 \%$ & $34.00 \%$ \\
\hline
\end{tabular}

Table 2. Recall Rate

\begin{tabular}{rrrr}
\hline & $50 \%: 50 \%$ & $70 \%: 30 \%$ & $80 \%: 20 \%$ \\
\hline 6 retrieval & $78.67 \%$ & $78.75 \%$ & $78.80 \%$ \\
8 retrieval & $80.00 \%$ & $80.75 \%$ & $81.20 \%$ \\
12 retrieval & $80.22 \%$ & $81.25 \%$ & $81.60 \%$ \\
\hline
\end{tabular}




\section{CONCLUSION}

Based on the tests performed, it can be concluded that the CBIR system using MSD (Micro Structure Descriptor) which based on android platform can run well or effectively. From the results of the research, the best system performance is obtained by dividing the data by $80 \%$ or 250 of training data and $20 \%$ or 50 test data, with 6 retrievals, yielding the precision rate of $65.67 \%$ and the recall rate of $78.80 \%$.

\section{REFERENCES}

[1] Haake, A., "The Role of Symmetry in Javanese Batik Patterns", Computers \& Mathematics with Applications, vol. 17, no. 4-6, pp. 815-826, 1989.

[2] Saddhono, K., et al., "The study of Philosophical Meaning of Batik and Kimono Motifs to Foster Collaborative Creative Industry”, Asian Social Science, vol. 10, no. 9, p. 52, 2014.

[3] W. Mashadi, et al., Batik Indonesia Mahakarya Penuh Pesona, Jakarta: Kaki- Langit Kencana, 2015.

[4] Banerjee, D., Journey of Textile Designs: A Case Study of Batik in Java and Santiniketan (Master's thesis), 2016.

[5] S. S. Samsi, Techniques, Motifs, Patterns Batik Yogya and Solo, Titian Foundation, 2011

[6] H. S. Doellah, Batik: The Impact of Time and Environment, Danar Hadi Solo, 2003.

[7] J. Achjadi, The Glory of Batik: The Danar Hadi Collection, 1st ed, Central Java, PT. Batik Danar Hadi, 2011.

[8] Minarno, A. E., and Suciati, N., "Batik Image Retrieval based on Color difference Histogram and Gray Level Co-Occurrence Matrix”, TELKOMNIKA (Telecommunication Computing Electronics and Control), vol. 12, no. 3, pp. 597-604, 2014.

[9] Minarno, A. E., et al., "Batik Image Retrieval based on Enhanced Micro-Structure Descriptor," in Computer Aided System Engineering (APCASE), 2014 Asia-Pacific Conference on. IEEE, 2014, pp. 65-70.

[10] Minarno, A. E., and Suciati, N., "Image Retrieval Using Multi Texton Co-Occurrence Descriptor", Journal of Theoretical \& Applied Information Technology, vol. 67, no. 1, 2014.

[11] Minarno, A. E., et al., "Image Retrieval Based on Multi Structure Co-occurrence Descriptor", TELKOMNIKA (Telecommunication Computing Electronics and Control), vol. 14, no. 3, pp. 1175-1182, 2016.

[12] Kurniawardhani, A., et al., "Efficient texture image retrieval of improved completed robust local binary pattern", In Advanced Computer Science and Information Systems (ICACSIS), 2016 International Conference on, pp. 492-497, IEEE, Oct 2016.

[13] Nurhaida, I., et al., "Texture Fusion for Batik Motif Retrieval System", International Journal of Electrical and Computer Engineering (IJECE), vol. 6, no. 6, p. 3174, 2016.

[14] Gudivada, V. N., and Raghavan, V. V., "Content based Image Retrieval Systems", Computer, vol. 28, no. 9, pp. 18-22, 1995.

[15] Rui, Y., et al., "Relevance Feedback: A Power Tool for Interactive Content-based Image Retrieval", IEEE Transactions on Circuits and Systems for Video Technology, vol. 8, no. 5, pp. 644-655, 1998.

[16] Smeulders, A. W., et al., "Content-based Image Retrieval at the end of the early years", IEEE Transactions on Pattern Analysis and Machine Intelligence, vol. 22, no. 12, pp.1349-1380, 2000.

[17] Shete, D. S., et al., "Content based Image Retrieval", International Journal of Emerging Technology and Advanced Engineering, vol. 2, no. 9, pp. 85-90, 2012.

[18] S. Nugroho and D. Utomo, "Rotation Invariant Indexing for Image using Zernike Moments and Rtree," TELKOMNIKA (Telecommunication Computing Electronics and Control), vol. 9, no. 2, pp. 335-340, 2013.

[19] G.-H. Liu and J.-Y. Yang, "Content-based Image Retrieval using Color difference Histogram," Pattern Recognition, vol. 46, no. 1, pp. 188-198, 2013.

[20] Liu, Guang-Hai, and Jing-Yu Yang, "Image Retrieval based on the Texton Co-occurrence Matrix", Pattern Recognition 41.12 (2008), pp. 3521-3527.

[21] G.-H. Liu, et al., "Image Retrieval based on Multi-texton Histogram," Pattern Recognition, vol. 43, no. 7, pp. 2380-2389, 2010.

[22] G.-H. Liu, et al., "Image Retrieval based on Micro-structure Descriptor," Pattern Recognition, vol. 44, no. 9, pp. 2123-2133, 2011.

[23] Minarno, A. E., et al., "Classification of Texture Using Multi Texton Histogram and Probabilistic Neural Network", In IOP Conference Series: Materials Science and Engineering, vol. 105, no. 1, p. 012022, IOP Publishing, Jan 2016.

[24] Julesz, B, "Textons, the Elements of Texture Perception, and their Interactions", Nature, vol. 290, no. 5802, pp. 91-97, PMID 7207603, March 1981. 